\title{
Validade da velocidade crítica para a determinação dos efeitos do treinamento no limiar anaeróbio em corredores de endurance
}

\author{
BS Denadai ${ }^{1}$, MJ Ortiz ${ }^{1}$, S Stella ${ }^{2}$, MT Mello ${ }^{2}$ \\ https://doi.org/10.5628/rpcd.03.01.16
}

\author{
${ }^{1}$ Laboratório de Avaliação da Performance Humana \\ UNESP, Rio Claro, Brasil \\ ${ }^{2}$ Centro de Estudos em Psicobiologia e Exercício - EPM \\ UNIFESP, São Paulo, Brasil
}

\section{RESUMO}

O objetivo deste estudo foi analisar a validade da velocidade crítica (VC) para estimar o limiar anaeróbio (LAn), antes e após dois diferentes programas de treinamento em atletas de endurance. Dezessete corredores (33,4 $\pm 4,4$ anos; $62,7 \pm 4,3$ $\mathrm{kg} ; 166,1 \pm 5,0 \mathrm{~cm}$ ) divididos em 2 grupos (G95\% - $\mathrm{N}=9$; G100\% $-\mathrm{N}=8$ ) realizaram os seguintes testes: 1 ) Tiros máximos de 1500 e $5000 \mathrm{~m}$ para a determinação da VC; 2) Testes para a determinação do consumo máximo de oxigênio $\left(\mathrm{VO}_{2}\right.$ max $)$ e sua respectiva velocidade $\left(\mathrm{vVO}_{2} \mathrm{max}\right)$, economia de corrida (EC), LAn e tempo de exaustão (Tlim) nas intensidades de 95 e $100 \% \mathrm{vVO}_{2} \max$. Os testes foram realizados antes e após 4 semanas de treinamento, o qual foi semelhante entre os grupos, exceto nos 2 dias de treinamento intervalado. Antes do treinamento, não houve diferença entre o LAn $(17,31 \pm 1,3$; $17,35 \pm 0,8 \mathrm{~km} / \mathrm{h})$ e a VC $(16,97 \pm 1,1 ; 17,44 \pm 0,9 \mathrm{~km} / \mathrm{h})$ para os G95\% e G100\%, respectivamente. Após o treinamento, houve aumento do LAn $(\mathrm{G} 95 \%=17,98 \pm 1,1 ; \mathrm{G} 100 \%=$ $18,11 \pm 0,6 \mathrm{~km} / \mathrm{h}$ ) para os dois grupos, e da VC para o G95\% $(\mathrm{G} 95 \%=17,25 \pm 1,0 ; \mathrm{G} 100 \%=17,63 \pm 0,7 \mathrm{~km} / \mathrm{h})$. Neste período, o LAn foi maior do que a VC para os dois grupos. Conclui-se que a validade da VC para estimar o LAn determinado com concentração fixa de lactato $(4 \mathrm{mM})$, pode ser dependente do período e/ou tipo de treinamento realizado pelos atletas de endurance.

Palavras-chave: corrida, velocidade crítica, limiar anaeróbio.

\author{
ABSTRACT \\ Validity of the critical velocity for determination of the training \\ effects on the anaerobic threshold in endurance runners.
}

The objective of this study was to analyze the validity of the critical velocity (CV) to evaluate the anaerobic threshold (AnT), before and after two different training programs in endurance athletes. Seventeen male runners $(33.4 \pm 4.4$ years; $62.7 \pm 4.3 \mathrm{~kg} ; 166.1 \pm 5.0 \mathrm{~cm})$ divided in two groups (G95\% $-N=9$ and $G 100 \%-N=8$ ) performed the following tests: 1) Maximum shots of 1500 and $5000 \mathrm{~m}$ for determination of the $\mathrm{CV}$; 2) Determination of the VO2max, the speed corresponding to VO2max ( $v V O 2 \max$ ), running economy (EC), AnT and time to exhaustion (Tlim) at the intensity of 95 and 100\% vVO2max. Tests were performed before and after 4 weeks of training, which was similar to both groups, except for the two days of interval training. Before training, there were no differences between AnT $(17.31 \pm 1.3 ; 17.35 \pm 0.8 \mathrm{~km} / \mathrm{h})$ and $C V(16.97 \pm 1.1 ; 17.44 \pm$ $0,9 \mathrm{~km} / \mathrm{h})$ for $\mathrm{G} 95 \%$ and $\mathrm{G100 \%}$, respectively. After training, there was an improvement in AnT (G95\% = $17.98 \pm 1.1 ; G 100 \%=$ $18.11 \pm 0.6 \mathrm{~km} / \mathrm{h}$ ) for the two groups, and in CV for G95\% (G95\% $=17.25 \pm 1.0 ; \mathrm{G} 100 \%=17.63 \pm 0.7 \mathrm{~km} / \mathrm{h}$ ). In this period, the AnT was higher than the $C V$ for the two groups. We can conclude that the validity of the $C V$ to evaluate the AnT determined with fixed blood lactate concentration $(4 \mathrm{mM})$, may be dependent on the period and/or type of training performed by endurance athletes.

Key Words: running, critical speed, anaerobic threshold. 


\section{INTRODUÇÃ̃O}

A determinação da máxima fase estável de lactato sanguíneo (MLACSS), definida como a máxima intensidade de exercício de carga constante onde ainda se observa equilíbrio entre a taxa de liberação e remoção do lactato sanguíneo, tem sido estudada por diversos pesquisadores $(2,14,25)$. A MLACSS parece ser o limite superior onde ainda se observa estabilidade nas respostas metabólicas e nas trocas gasosas pulmonares (23), sendo frequentemente indicada para a prescrição do treinamento aeróbio, particularmente em atletas $(15,18)$.

A identificação da MLACSS apresenta em princípio uma desvantagem, pois exige a realização de 4-6 séries de exercícios de carga constante, com aproximadamente 30 min de duração, obrigando a vinda do atleta ao laboratório por vários dias. Para tentar retirar esta desvantagem, Heck et al. (9) propuseram a identificação da MLACSS com base em um único protocolo de cargas progressivas, empregando uma concentração fixa de $4 \mathrm{mM}$ de lactato sanguíneo. Embora possa ser vantajoso, diversos autores criticam este critério, pois pode-se encontrar uma variabilidade relativamente grande nestas concentrações, tanto entre os indivíduos (27) como também em função do tipo de exercício (2). Entretanto, muitos estudos realizados especificamente em corredores têm confirmado que a MLACSS corresponde em média a $4 \mathrm{mM}(14,25)$, mesmo em atletas mais jovens (1).

Por outro lado, independentemente destes aspectos, a determinação do lactato sanguíneo requer o uso de equipamentos sofisticados que nem sempre estão disponíveis, restringindo sua utilização a um pequeno número de atletas de elite. Deste modo, alguns estudos têm procurado validar outros índices que possam ser utilizados na avaliação aeróbia, sem envolver coleta de sangue e equipamentos caros (7). Um destes índices é a potência crítica ou velocidade crítica (VC), definida como a mais alta intensidade de exercício que teoricamente pode ser mantida por um longo período de tempo sem exaustão. Seu conceito é baseado na relação hiperbólica entre intensidades pré-determinadas e seus respectivos tempos de exaustão. Especificamente para a corrida, Kranenburg \& Smith (16) e Ortiz et al. (22) verificaram que a $\mathrm{VC}$ pode ser obtida pela regressão linear entre distâncias fixas e seus respectivos tempos, sendo que a VC corresponde ao coeficiente angular da reta obtida.

Embora alguns estudos tenham verificado que a VC é um bom preditor da performance em corridas de longa duração $(10 \mathrm{~km})(11,16)$, não foram encontradas informações sobre a validade da VC em predizer a resposta do lactato sanguíneo, particularmente antes e após um programa de treinamento. Com isso, o objetivo deste estudo foi analisar a validade da VC para estimar o limiar anaeróbio (LAn), antes e após dois diferentes programas de treinamento em atletas de endurance.

\section{MATERIAL E MÉTODOS \\ Sujeitos}

Participaram deste estudo 17 corredores $(33,4 \pm 4,4$ anos; $62,7 \pm 4,3 \mathrm{~kg} ; 166,1 \pm 5,0 \mathrm{~cm} ; 6,3 \pm 2,9 \%$ de gordura corporal e; 7,2 $\pm 4,9$ anos de treinamento) bem treinados nas provas de fundo do atletismo. Todos os corredores treinavam 6 dias por semana, com um volume semanal que oscilava entre 70 a 90 $\mathrm{km}$ durante o período de preparação específica. O estudo foi aprovado pelo Comitê de Ética da instituição e todos os participantes conheceram os procedimentos do experimento e suas implicações (riscos e benefícios), através de um termo de consentimento escrito e explicado.

\section{Delineamento experimental}

No início do experimento, todos participantes estavam cumprindo a $4 \underline{\mathrm{a}}$ semana do período de preparação específica da periodização do treinamento. $\mathrm{O}$ experimento foi desenvolvido durante 8 semanas, sendo estas, subdivididas em 4 diferentes fases (Figura 1).

\begin{tabular}{lrrrrrrrr} 
Semanas & 1 & 2 & 3 & 4 & 5 & 6 & 7 & 8 \\
\hline Períodos & Pré-teste & \multicolumn{1}{l}{ Treinamento } & Polimento & Pós-teste
\end{tabular}

Figura 1 - Duração do experimento e suas respectivas fases.

Nas semanas do pré (1므 e $2 \underline{a})$ e pós-teste (7aa e $8 \underline{a})$ ocorreu uma redução do volume de treinamento nas sessões intervaladas, devido ao início das competi- 
ções simuladas e avaliações em laboratório. Inicialmente, os atletas realizaram a competição simulada nas distâncias de 1500 (meio fundo) e 5000 metros (fundo).

Posteriormente, iniciaram-se os testes em laboratório, para a determinação do consumo máximo de oxigênio $\left(\mathrm{VO}_{2} \mathrm{max}\right)$, da velocidade correspondente ao $\mathrm{VO}_{2} \max$ ( $\left.\mathrm{vVO}_{2} \mathrm{max}\right)$, do LAn, da economia de corrida (EC) e do tempo de exaustão (Tlim) realizado na $\mathrm{vVO}_{2}$ max. Entre cada teste, foi respeitado um intervalo de no mínimo 48 horas, em que cada corredor foi instruído a realizar somente um treino de baixa intensidade por um período máximo de 30-40 minutos.

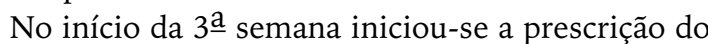
treinamento baseado nos resultados obtidos nos testes de laboratório, tendo a duração total de 4 semanas (24 dias úteis de treino). Nesta etapa os atletas foram divididos aleatoriamente em dois grupos (G95\% - N = 9 e $\mathrm{G} 100 \%-\mathrm{N}=8)$. Os grupos realizaram ao longo desta fase o mesmo tipo de treinamento, exceto nos dois dias de treinamento intervalado, cuja velocidade de execução dos tiros (\%vVO 2 max) e duração destes (\%Tlim) foram diferenciadas, embora a metragem total percorrida no treino intervalado tenha sido semelhante entre os grupos. Neste período, então, os atletas realizaram 2 sessões de treinamento intervalado, mais 1 sessão com velocidade correspondente ao LAn (40 min), além de mais 3 sessões de treinamento contínuo (60 min a $70 \% \mathrm{VO}_{2} \max$ ), que foram intercaladas entre os dias de treino intervalado, totalizando 6 sessões semanais.

Para o G95\% a velocidade de execução dos tiros durante o treinamento intervalado foi de $95 \%$ $\mathrm{vVO}_{2}$ max, com duração de $60 \%$ Tlim obtido a $95 \% \mathrm{vVO}_{2} \max$. A recuperação ativa entre os tiros foi realizada a $50 \% \mathrm{vVO}_{2}$ max e com uma duração de $30 \%$ Tlim, sendo o esforço repetido por 4 vezes. Para o G100\%, a velocidade de execução dos tiros durante o treinamento intervalado foi de $100 \% \mathrm{vVO}_{2}$ max, com duração de $60 \%$ Tlim obtido a $100 \% \mathrm{vVO}_{2}$ max. A recuperação ativa entre os tiros foi realizada a $50 \% \mathrm{vVO}_{2}$ max e com uma duração de $60 \%$ Tlim, sendo o esforço repetido por 5 vezes. Na $7 \underline{\text { a }}$ semana, houve redução do volume dos tiros e aumento da intensidade nas 2 sessões intervaladas. As demais sessões de treinamento foram mantidas.
Determinação do $\mathrm{VO}_{2} \max , \mathrm{vVO}_{2} \max$ e LAn.

$\mathrm{O} \mathrm{VO}_{2}$ max, a $\mathrm{VVO}_{2}$ max e o LAn foram determinados a partir de um teste incremental em uma esteira rolante (LIFE FITNESS 9800), com velocidade inicial de $12 \mathrm{~km} / \mathrm{h}$ e incrementos de $2 \mathrm{~km} / \mathrm{h}$ a cada 3,5 minutos até atingir $80 \%$ da velocidade individual de corrida nos $1500 \mathrm{~m}$. Posteriormente, os incrementos foram de $1 \mathrm{~km} / \mathrm{h}$ até a exaustão voluntária. Entre cada estágio houve uma pausa de 30 segundos para a coleta de $25 \mathrm{ml}$ de sangue do lóbulo da orelha (21). A inclinação da esteira foi mantida fixa em $1 \%$, já que esta condição reflete mais precisamente o custo energético da corrida em ambientes abertos (13). Durante os testes, os gases expirados foram avaliados no modo respiração-respiração (SENSOR MEDICS - MMC), com registros a cada 10 segundos. A maior média de 2 valores consecutivos registrados a cada 10 s chamamos de $\mathrm{VO}_{2} \max$. $\mathrm{A} v \mathrm{vO}_{2}$ max foi a velocidade mínima em que o $\mathrm{VO}_{2}$ max foi alcançado e mantido por ao menos 1 min. Se o $\mathrm{VO}_{2}$ max alcançado durante o estágio não fosse sustentado por 1 min, a velocidade do estágio anterior era assumida como a vVO 2 max (4).

O lactato sanguíneo foi determinado por um método eletroquímico (YSL 2300), e o LAn foi identificado como a velocidade correspondente a uma concentração fixa de $4 \mathrm{mM}$ (9).

\section{Teste para determinar a economia de corrida (EC) e o tempo de exaustão (Tlim)}

Os corredores realizaram um aquecimento por $7 \mathrm{~min}$ a $12 \mathrm{~km} / \mathrm{h}$, seguido por um descanso de $3 \mathrm{~min}$ e, posteriormente, correram por mais $8 \mathrm{~min}$ a $14 \mathrm{~km} / \mathrm{h}$. $\mathrm{O}$ consumo de oxigênio $\left(\mathrm{VO}_{2}\right)$ foi medido entre o $6^{\circ}$ e $7^{\circ} \mathrm{min}$ a $14 \mathrm{~km} / \mathrm{h}$, servindo como referência para a EC do atleta, que foi definida como a relação entre o $\mathrm{VO}_{2}$ e a velocidade de corrida (6).

Imediatamente após o $8^{\circ}$ min de corrida submáxima, num prazo de 30 a 45 segundos, a velocidade da esteira era ajustada à intensidade correspondente a $95 \%(\mathrm{G} 95 \%)$ ou $100 \%(\mathrm{G} 100 \%) \mathrm{vVO}_{2} \max$, iniciando-se a corrida até a exaustão voluntária (4).

\section{Determinação da Velocidade Crítica (VC)}

Conforme proposto por Hill (10), simulamos uma competição nas distâncias de 1500 e 5000 metros em pista de atletismo emborrachada de 400 metros, em dias diferentes dentro da mesma semana. A VC 
foi determinada através do coeficiente angular (b) da reta de regressão linear entre as distâncias e os respectivos tempos.

\section{Análise estatística}

Os dados estão expressos com média \pm desvio padrão (DP). A ANOVA TWO-WAY para dados repetidos, complementada pelo teste de Tukey, foi empregada para comparar o LAn e a VC antes e após o período de treinamento. $O$ teste de correlação de Pearson foi utilizado para verificar a correlação entre o LAn e a VC. O teste $t$ de Student para dados pareados foi utilizado para comparar as demais variáveis antes e após o período de treinamento Em todos os testes foi adotado um nível de significância de $\mathrm{p} \leq 0,05$.

\section{RESULTADOS}

A Tabela 1 mostra os efeitos do treinamento sobre a $\mathrm{vVO}_{2} \max , \mathrm{VO}_{2} \max$ e EC para os atletas do $\mathrm{G} 95 \%$ e $\mathrm{G} 100 \%$. O treinamento só determinou aumento da $\mathrm{vVO}_{2}$ max e da EC para os atletas do G100\%.

Tabela 1 - Valores médios + DP da velocidade mínima em que ocorreu o maior consumo de oxigênio [ $\mathrm{VVO}_{2} \mathrm{max}$ ), do consumo máximo de oxigênio $\left(\mathrm{VO}_{2} \mathrm{max}\right)$ e da economia de corrida $[\mathcal{E C}]$ do grupo I [95\% $\mathrm{VVO}_{2}$ max] e do grupo II (100\% $\mathrm{VVO}_{2}$ max] antes (Pré] e depois [Pós] do programa de treinamento.

\begin{tabular}{lcccc} 
& \multicolumn{2}{c}{$\mathrm{GI}(\mathrm{n}=9)$} & \multicolumn{2}{c}{$\mathrm{GII}(\mathrm{n}=8)$} \\
& Pré & Pós & Pré & Pós \\
\hline $\mathrm{VVO}_{2} \mathrm{max}(\mathrm{km} / \mathrm{h})$ & $19,00 \pm 1,0$ & $19,22 \pm 0,9$ & $18,30 \pm 0,5$ & $19,06 \pm 1,0^{*}$ \\
$\mathrm{VO}_{2} \mathrm{max}(\mathrm{ml} / \mathrm{kg} / \mathrm{min})$ & $59,05 \pm 6,0$ & $58,97 \pm 5,7$ & $59,98 \pm 6,0$ & $58,35 \pm 5,4$ \\
$\mathrm{EC}(\mathrm{ml} / \mathrm{kg} / \mathrm{min})$ & $37,25 \pm 2,2$ & $36,30 \pm 1,7$ & $37,66 \pm 3,9$ & $36,27 \pm 3,7^{*}$ \\
& $*(p<0,05)$ em relação ao pré-teste dentro do mesmo grupo.
\end{tabular}

As velocidades correspondentes ao LAn e a VC, antes e após o programa de treinamento, estão apresentadas na Tabela 2. Como pode ser observado, durante o pré-teste não foram observadas diferenças significantes entre o LAn e a VC para os dois grupos estudados. Após o programa de treinamento, houve melhora significante do LAn para os dois grupos, e da VC para o G95\%. Neste período, o LAn foi significantemente maior do que a VC para os dois grupos estudados.

\begin{tabular}{|c|c|c|c|c|}
\hline GRUPO & LAn (Pré) [km/h] & LAn (Pós] (km/h] & VC (Pré) (km/h) & VC [Pós] $(\mathrm{km} / \mathrm{h}]$ \\
\hline$I(n=9)$ & $17,31 \pm 1,3$ & $17,98 \pm 1,1 *$ & $16,97 \pm 1,1$ & $17,25 \pm 1,0 * \dagger$ \\
\hline$\|(n=8)$ & $17,35 \pm 0,8$ & $18,11 \pm 0,6^{*}$ & $17,44 \pm 0,9$ & $17,63 \pm 0,7 \dagger$ \\
\hline
\end{tabular}

Houve correlação significante entre a velocidade do LAn e a VC do G95\% ( $r=0,90$ e $r=0,91)$ e G100\% ( $r=$ 0,84 e $r=0,80$ ) na condição pré-treinamento e pós-treinamento, respectivamente.

A Tabela 3 apresenta o tempo dos atletas nos 1500 e 5000 metros, antes e após o programa de treinamento. Para o G100\% houve redução significante do tempo de 1500 e 5000 metros. No G95\% houve melhora significante apenas nos 5000 metros. 


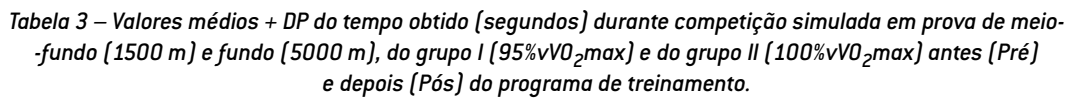

\begin{tabular}{lcccc} 
GRUPO & 1500 (Pré) & 1500 (Pós) & 5000 (Pré) & 5000 (Pós) \\
\hline I $(n=9)$ & $271,1 \pm 13,5$ & $269,0 \pm 13,4$ & $1016,0 \pm 61,8$ & $1001,0 \pm 56,9^{*}$ \\
II $(n=8)$ & $270,7 \pm 8,7$ & $265,5 \pm 8,4^{*}$ & $994,7 \pm 44,8$ & $981,0 \pm 39,6^{*}$ \\
& $*(p<0,05)$ em relação ao pré-teste dentro do mesmo grupo.
\end{tabular}

\section{DISCUSSÃO}

O principal objetivo deste estudo foi analisar a validade da VC em estimar o LAn, antes e após dois diferentes programas de treinamento, em atletas de endurance. Antes do programa de treinamento, a VC apresentou boa validade para a determinação do LAn. Após o treinamento, entretanto, a VC, para os dois grupos estudados, não apresentou a mesma sensibilidade do LAn para identificar as adaptações determinadas pelo treinamento.

Estudos realizados recentemente têm verificado que a inclusão de 1 a 2 sessões semanais de treinamento intervalado de alta intensidade (90 - 100 $\left.\% \mathrm{VO}_{2} \max \right)$, durante períodos relativamente curtos (4 semanas), são eficientes para a melhora de índices fisiológicos $\left(\mathrm{VO}_{2} \max , \mathrm{vVO}_{2} \max , \mathrm{LAn}\right.$ e $\left.\mathrm{EC}\right)$, e da performance aeróbia $(4,17)$.

Os programas de treinamento empregados neste estudo foram elaborados com base nos dados encontrados por Billat et al. (4). Os resultados obtidos pelo G100\% em nosso estudo são consistentes com os verificados por Billat et al. (4), mostrando que a $\mathrm{vVO}_{2}$ max pode ser aumentada a partir da melhora da EC, sem modificação do $\mathrm{VO}_{2}$ max. Podemos constatar ainda que estas modificações parecem ser dependentes do treinos intervalados a $100 \% \mathrm{VO}_{2} \max$, já que o G95\% não apresentou estas adaptações. Já em relação à melhora do LAn, os dois programas de treinamento se mostraram eficientes, não nos permitindo atribuir principalmente às sessões intervaladas e nem às intensidades empregadas nos mesmos, as adaptações que modificaram a resposta do lactato sanguíneo ao exercício incremental.

Os efeitos do treinamento sobre a performance também foram diferentes entre os grupos, sendo entretanto consistentes com as modificações encontradas nos diferentes índices fisiológicos. Estudos realizados em corredores com níveis similares de rendimento mostram que a performance de média e longa duração (> $5000 \mathrm{~m}$ ) é mais dependente do LAn (5), enquanto em provas aeróbias de curta duração (1500 - $3000 \mathrm{~m})$ a $\mathrm{vVO}_{2}$ max parece explicar mais as variações da performance (3). Como o G100\% aumentou a $\mathrm{vVO}_{2}$ max e o LAn, poder-se-ia realmente esperar a melhora dos 1500 e 5000 m. Por outro lado, o G95\% modificou só o LAn e provavelmente em função disto a performance só melhorou nos $5000 \mathrm{~m}$. O emprego de uma determinada concentração fixa de lactato obtido durante um teste incremental para determinar indiretamente a MLACSS tem gerado muito controvérsia entre os pesquisadores. Embora este critério possa reduzir o tempo e os custos do processo de avaliação, muitos autores o criticam, pois fatores como a variabilidade individual (27), o estado nutricional (19) e o tipo de exercício (2) podem influenciar os valores de lactato correspondentes a MLACSS determinada em exercícios de carga constante. Deve-se ressaltar, entretanto, que muitos estudos realizados especificamente em corredores têm confirmado que a MLACSS corresponde em média a $4 \mathrm{mM}(14,25)$, mesmo em atletas mais jovens (1). Independentemente disto, o LAn identificado com concentração fixa tem-se mostrado válido para identificar as adaptações provocadas pelo treinamento (7).

A validade da PC ou VC em predizer a performance aeróbia $(11,16)$ ou os limiares de lactato $(26,28)$ ou ventilatórios (20) tem sido bastante investigada, particularmente para o ciclismo estacionário e a natação. Para a corrida, Hughson et al. (11) foram os primeiros a aplicar o conceito de VC durante o exercício em esteira rolante, encontrando uma correlação 
moderada $(r=0,67)$ com a performance na prova de $10 \mathrm{~km}$. Posteriormente, Kranenburg \& Smith (16) verificaram que a determinação da VC em teste de pista (distância $\mathrm{x}$ tempo) é válida, apresentando valores semelhantes e altamente correlacionados com a VC determinada na esteira rolante (velocidades pré-estabelecidas $\mathrm{x}$ tempo de exaustão).

Confirmando estes resultados, verificamos em nosso laboratório (22) que a VC determinada em pista, como sugerido por Hill (10) com apenas duas distâncias (1500 e 5000 m), é válida para determinar a VC obtida em esteira, em corredores de endurance. Antes do período de treinamento, verificamos que a VC apresentou boa validade para estimar o LAn em corredores de endurance. Estes resultados são semelhantes aos de Wakayoshi et al. (28), que verificaram que a VC foi válida para determinar o LAn (4 mM) em um grupo de nadadores adultos (16-24 anos) e bem treinados. Posteriormente, Wakayoshi et al.

(29) também encontraram uma alta correlação $(r=$ 0,91 ) entre a VC e o LAn, verificando ainda que a VC correspondeu à MLACSS em um grupo de nadadores treinados colegiais (18 - 20 anos).

Após o período de treinamento, entretanto, a validade da VC pode ser questionada, pois a mesma não apresentou a mesma sensibilidade do LAn para detectar os efeitos do treinamento, embora os índices tenham permanecido bem correlacionados. Poucos estudos têm analisado os efeitos do treinamento sobre a PC/VC, principalmente em atletas que já se encontravam treinando. Jenkins e Quigley (12) verificaram em um grupo de indivíduos ativos, porém sem treinamento sistemático ao início do experimento, que o treinamento contínuo de intensidade sub-máxima (3 dias/semana - 8 semanas) aumentou a PC (30\%) e a intensidade máxima que podia ser sustentada por 40 min (28\%). Gaesser e Wilson (8) verificaram que os treinamentos contínuos de baixa intensidade (40 min a $50 \% \mathrm{VO}_{2} \max , 3$ dias/semana) ou intermitente de alta intensidade ( $10 \times 2 \mathrm{~min}$ a $100 \% \mathrm{VO}_{2}$ max, 3 dias/semana) determinaram aumento da PC de modo bem semelhante (15\%). Assim, a ausência (G100\%) ou a menor (G95\%) sensibilidade da VC podem ser atribuídas em parte ao maior nível inicial de condicionamento dos nossos voluntários, pois em nosso estudo utilizamos indivíduos treinados, o que potencialmente diminui a sensibilidade aos efeitos do treinamento.

Este aspecto não explica completamente as diferentes sensibilidades do LAn e da VC nos dois grupos analisados, sendo entretanto semelhante ao encontrado por Poole et al. (24). Poole et al. (24) realizaram o único estudo que avaliou simultaneamente os efeitos de 7 semanas de treinamento $(10 \times 2$ min a $105 \% \mathrm{VO}_{2}$ max, 3 dias/semana) sobre a PC e um índice que determinou indiretamente a resposta do lactato ao exercício incremental (limiar ventilatório), porém em indivíduos sem treinamento prévio.

Similarmente ao nosso estudo, Poole et al. (24) verificaram que o limiar ventilatório (24\%) apresentou maior sensibilidade aos efeitos do treinamento do que a PC (15\%). Deste modo, os mecanismos que determinam as adaptações na resposta do lactato, podem não ser exatamente os mesmos que modificam a VC, podendo existir diferentes comportamentos nestes índices em função do treinamento físico. Um aspecto que merece destaque em nosso estudo é o fato das performances nos 1500 e $5000 \mathrm{~m}$ terem melhorado significantemente no G100\%, enquanto a VC permaneceu constante. Como a VC foi determinada como o coeficiente angular da relação distância $x$ tempo, é provável que os percentuais de melhora tenham sido diferentes entre as distâncias (1500 e $5000 \mathrm{~m}$ ) e também entre os indivíduos. Este aspecto acabou determinando que o coeficiente angular (VC) tenha apresentado comportamentos diferentes entre os indivíduos (diminuição, manutenção ou aumento), fazendo com que a VC não se modificasse significantemente. Para o G95\%, a performance nos $1500 \mathrm{~m}$ não se modificou, enquanto nos $5000 \mathrm{~m}$ houve melhora significante. Deste modo, poder-se-ia realmente esperar aumento do coeficiente angular na relação distância x tempo (VC), como de fato ocorreu. Finalmente, não podemos descartar completamente um possível efeito dos níveis de glicogênio muscular (depleção) sobre o LAn, principalmente no período de pós-treinamento. Maassen e Busse (19) verificaram que a intensidade correspondente ao LAn que utiliza concentração fixa de lactato, pode ser superestimada, caso os indivíduos estejam com depleção de glicogênio muscular. Ressalta-se, entretanto, que procuramos evitar este fenômeno com a inclusão da semana de polimento e a redução da sobrecarga na semana dos testes em laboratório. 
De qualquer modo, são necessários mais estudos que analisem a relação entre a VC e a resposta de lactato sanguíneo, envolvendo atletas em diferentes fases e/ou tipo de treinamento, principalmente que possam determinar diretamente a MLACSS, para que possamos obter informações mais definitivas. Com base nos resultados deste estudo, podemos concluir que a validade da VC para estimar o LAn, determinado com concentração fixa de lactato (4 $\mathrm{mM}$ ), pode ser dependente do período e/ou tipo de treinamento realizado pelos atletas de endurance.

\section{CORRESPONDÊNCIA}

\section{B.S. Denadai}

Laboratório de Avaliação da Performance Humana IB, UNESP

Av. 24 A, 1515, Bela Vista - Rio Claro - SP

CEP - 13506-900 Brasil

bdenadai@rc.unesp.br 


\section{REFERÊNCIAS BIBLIOGRÁFICAS}

1 Ascensão AA, Santos P, Magalhães J, Oliveira J, Maia J, Soares J (2001). Concentrações sanguíneas de lactato (CSL) durante uma carga constante a uma intensidade correspondente ao limiar aeróbio-anaeróbio em jovens atletas. Rev Paul Educa Fis 15: 186-194

2 Beneke R, Von Duvillard SP (1996). Determination of maximal lactate steady state response in selected sports events. Med Sci Sports Exerc 28: 241-246

3 Billat V, Beillot J, Jan J, Rochcongar P, Carre F (1996) Gender effect on the relationship of time limit at $100 \%$ $\mathrm{O}_{2}$ max with other bioenergetic characteristics. Med. Sci. Sports Exerc 28: 1049-1055

4 Billat V, Flechet B, Petit B, Muriaux G, Koralsztein JP (1999). Interval training at $\mathrm{VO}_{2}$ max: effects on aerobic performance and overtraining markers. Med Sci Sports Exerc 3: 156-163

5 Coyle EF (1995). Integration of the physiological factors determining endurance performance ability. Exerc Sport Sci Rev 23: 25-63

6 Daniels J, Daniels N (1992). Running economy of elite male and elite female runners. Med Sci Sports Exerc 24 : 483-9

7 Denadai BS (2000). Avaliação aeróbia: Determinação indireta da resposta do lactato sanguíneo. Rio Claro: Motrix.

8 Gaesser GA, Wilson LA (1988). Effects of continuous and interval training on the parameters of the power-endurance time relationship for high-intensity exercise. Int J Sports Med $9: 417-21$

9 Heck H, Mader A, Hess G, Mucke S, Muller R, Hollmann W (1985). Justification of the $4 \mathrm{mmol} / \mathrm{l}$ lactate threshold. Int J Sports Med $6: 117-130$

10 Hill DW (2001). Aerobic and anaerobic contributions in middle distance running events. Motriz 7: 63-67

11 Hughson RL, Orok CJ, Staudt LE (1984). A high velocity treadmill running test to assess endurance running potential. Int J Sports Med $5: 23-25$

12 Jenkins DG, Quigley BM (1992). Endurance training enhances critical power. Med Sci Sports Exerc 24 : 1283-1289

13 Jones AM, Doust JH (1996). A 1\% treadmill grade most accurately reflects the energetic cost of outdoor running. $J$ Sport Sci 14: 321-327

14 Jones AM, Doust JH (1998). The validity of the lactate minimum test for determination of the maximal of the maximal lactate steady state. Med Sci Sports Exerc 30 : 1304-1313

15 Kindermann W, Simon G, Keul J (1979). The significance of the aerobic-anaerobic transition for the determination of work load intensities during endurance training. Eur J Appl Physiol 42: 25-34

16 Kranenburg K, Smith D (1996). Comparison of critical speed determined from track running and treadmill tests in elite runners. Med Sci Sports Exerc 28: 614-618

17 Lindsay FH, Hawley JA, Myburgh KH, Schomer HH, Noakes TD, Dennis SC (1996). Improved athletic performance in highly trained cyclists after interval training. Med Sci Sports Exerc 28: 1427-1434

18 Londeree BR (1997). Effect of training on lactate/ventilatory thresholds: a meta-analysis. Med Sci Sports Exerc 29 : 837-843

19 Maassen N, Busse MW (1989). The relationship between lactic acid and work load: a measure for endurance capa- city or an indicator of carbohydrate deficiency?. Eur J Appl Physiol 58: 728-737

20 Moritani TA, Nagata HÁ, Devries HÁ, Muro M (1981). Critical power as a measure of physical work capacity and anaerobic threshold. Ergonomics 24: 339-350

21 Morton RH, Billat V (2000). Maximal endurance time at $\mathrm{VO}_{2}$ max. Med Sci Sports Exerc 32: 1496-1504

22 Ortiz MJ, Denadai BS (2002). Efeito do treinamento sobre o limiar anaeróbio, velocidade crítica determinada em pista de atletismo e esteira, e performance de corredores de fundo. In WW Moreira (ed.) Coletâneas do II Congresso Científico Latino-Americano. Piracicaba: UNIMEP, 292-297

23 Poole DC, Ward SA, Gardner GW, Whipp BJ (1988). Metabolic and respiratory profile of the upper limit for prolonged exercise in man. Ergonomics 31: 1265-1279

24 Poole DC, Ward SA, Whipp BJ (1990). The effects of training on the metabolic and respiratory profile of highintensity cycle ergometer exercise. Eur J Appl Physiol Occup Physiol 59: 421-429

25 Simões HG, Campbell CSG, Kokubun E, Denadai BS, Baldissera V (1999). Blood glucose responses in humans mirror lactate responses for individual anaerobic threshold and for lactate minimum in track tests. Eur J Appl Physiol 80: $34-40$

26 Smith C, Jones A (2001). The relationship between critical velocity, maximal lactate steady state velocity and lactate turnpoint velocity in runners. Eur J Appl Physiol 85: 19-26

27 Stegmann H, Kindermann W, Schnabel A (1981). Lactate kinetics and individual anaerobic threshold. Int J Sports Med 2:160-5

28 Wakayoshi K, Ilkuta K, Yoshida T, Udo M, Moritani T, Mutoh Y (1992). Determination and validity of critical velocity as an index of swimming performance in the competitive swimmer. Eur J Appl Physiol 64: 153-7

29 Wakayoshi K, Yoshida T, Udo M, Harada T, Moritani T (1993). Does critical swimming velocity represent exercise intensity at maximal lactate steady-state? Eur J Appl Physiol 66: 90-5. 